\title{
Positive Periodic Solutions in a Discrete Time Three Species Competition System
}

\author{
Changjin $X u^{1}$ and Yusen $W u^{2}$ \\ ${ }^{1}$ Guizhou Key Laboratory of Economics System Simulation, Guizhou University of Finance and Economics, Guiyang 550004, China \\ ${ }^{2}$ Department of Mathematics and Statistics, Henan University of Science and Technology, Luoyang 471003, China
}

Correspondence should be addressed to Changjin Xu; xcj403@126.com

Received 11 December 2012; Accepted 27 January 2013

Academic Editor: Zhijun Liu

Copyright (c) 2013 C. Xu and Y. Wu. This is an open access article distributed under the Creative Commons Attribution License, which permits unrestricted use, distribution, and reproduction in any medium, provided the original work is properly cited.

A periodic discrete time three species competition system is investigated. With the aid of differential equations with piecewise constant arguments, a discrete analogue of continuous nonautonomous three species competition system is proposed. By using Gaines and Mawhin's continuation theorem of coincidence degree theory, sufficient conditions for the existence of positive periodic solutions of the model are obtained.

\section{Introduction}

During the past decades, the dynamical properties of competitive populations have received great attention from both theoretical and mathematical biologists due to their universal prevalence and importance. Numerous excellent results have been reported for a lot of different continuous or impulsive competitive models. For example, Kuang [1] analyzed the permanent coexistence of the following delayed three species competition system:

$$
\begin{gathered}
\frac{d x_{1}}{d t}=x_{1}(t)\left[1-x_{1}(t)-\int_{-\infty}^{t} K(s-t) x_{2}(s) d s\right. \\
\left.-\int_{-\infty}^{t} L(s-t) x_{3}(s) d s\right], \\
\frac{d x_{2}}{d t}=x_{2}(t)\left[1-x_{2}(t)-\int_{-\infty}^{t} L(s-t) x_{1}(s) d s\right. \\
\left.-\int_{-\infty}^{t} K(s-t) x_{3}(s) d s\right],
\end{gathered}
$$

$$
\begin{aligned}
\frac{d x_{2}}{d t}=x_{3}(t)[ & 1-x_{3}(t)-\int_{-\infty}^{t} K(s-t) x_{1}(s) d s \\
& \left.-\int_{-\infty}^{t} L(s-t) x_{2}(s) d s\right],
\end{aligned}
$$

where $x_{i}(t)(i=1,2,3)$ stands for the density of competing species at time $t$. For the biological meaning of model (1), one can see [1]. Tang et al. $[2,3]$ presented sufficient conditions for the existence and global attractivity of positive periodic solutions of the following periodic $n$-species Lotka-Volterra competition system with delays

$$
\dot{x}_{i}(t)=x_{i}(t)\left[r_{i}(t)-\sum_{j=1}^{n} a_{i j}(t) x_{j}\left(t-\tau_{i j}(t)\right)\right] .
$$

Bohner et al. [4] focused on the existence of periodic solutions in a predator-prey and competition dynamic systems, Pao [5] considered the global asymptotic stability of LotkaVolterra competition systems with diffusion and time delays, and Gopalsamy and Weng [6] made a detailed analysis on the global attractivity for a competition system with feedback controls. For more related work, one can see [7-10]. 
In 2011, Zhu and Lu [11] investigated the following delayed three species competitive system:

$$
\begin{aligned}
\frac{d x_{1}}{d t}=x_{1}(t)[ & r_{1}(t)-a_{11}(t) x_{1}(t) \\
& -a_{12}(t) \int_{-\infty}^{t} K_{12}(s-t) x_{2}(s) \mathrm{d} s \\
& \left.-a_{13}(t) \int_{-\infty}^{t} K_{13}(s-t) x_{3}(s) d s\right],
\end{aligned}
$$$$
\frac{d x_{2}}{d t}=x_{2}(t)\left[r_{2}(t)-a_{22}(t) x_{2}(t)\right.
$$$$
-a_{21}(t) \int_{-\infty}^{t} K_{21}(s-t) x_{1}(s) d s
$$$$
\left.-a_{23}(t) \int_{-\infty}^{t} K_{23}(s-t) x_{3}(s) d s\right],
$$$$
\frac{d x_{3}}{d t}=x_{3}(t)\left[r_{3}(t)-a_{33}(t) x_{3}(t)\right.
$$$$
-a_{31}(t) \int_{-\infty}^{t} K_{31}(s-t) x_{1}(s) d s
$$$$
\left.-a_{32}(t) \int_{-\infty}^{t} K_{32}(s-t) x_{2}(s) d s\right],
$$

where $x_{i}(t)(i=1,2,3)$ stands for the density of competing species at time $t, r_{i}, a_{i j} \in C(R,[0,+\infty))(i, j=1,2,3)$ are $\omega$-periodic functions $(\omega>0)$, and $K_{i j}(i, j=1,2,3)$ is a nonnegative function in $L_{1}(-\infty, 0]$ with

$$
\begin{gathered}
\bar{r}_{i}=\frac{1}{\omega} \int_{0}^{\omega} r_{i}(s) d s>0, \quad i=1,2,3, \\
K_{i j}=\int_{-\infty}^{0} K_{i j}(\theta) d \theta>0, \\
a_{i j}^{K}=\frac{1}{\omega} \int_{0}^{\omega} a_{i j}(\sigma) d \sigma \int_{-\infty}^{\sigma} K_{i j}(s-\sigma) d s>0 .
\end{gathered}
$$

For more details about the model, one can see [11]. By applying the theory of coincidence degree theory, Zhu and $\mathrm{Lu}$ [11] established the existence of positive periodic solution for system (3).

Numerous researchers have argued that discrete time models governed by difference equations are more appropriate to describe the dynamics relationship among populations than continuous ones when the populations have nonoverlapping generations. Moreover, discrete time models can also provide efficient models of continuous ones for numerical simulations. Therefore, it is reasonable and interesting to study discrete time systems governed by difference equations. Recently, a great deal of work has been devoted to this topics; see [12-19]. The principle purpose of this paper is to propose a discrete analogue of system (3) and study the effect of the periodicity of the ecological and environmental parameters on the dynamics of discrete time three species competition system.
The remainder of the paper is organized as follows. In Section 2, with the help of differential equations with piecewise constant arguments, we first propose a discrete analogue of system (3), modelling the dynamics of time nonautonomous competing system where populations have nonoverlapping generations. In Section 3, based on the coincidence degree and the related continuation theorem, sufficient conditions for the existence of positive solutions of difference equations are given.

\section{Discrete Analogue of System (3)}

There are several different ways of deriving discrete time version of dynamical systems corresponding to continuous time formulations. One of the ways of deriving difference equations modelling the dynamics of populations with nonoverlapping generations that we will use in the following is based on appropriate modifications of models with overlapping generations. For more details about the approach, we refer to $[17,20]$.

Next, we will discretize the system (3). Assume that the average growth rates in system (3) change at regular intervals of time; then we can obtain the following modified system:

$$
\begin{aligned}
\frac{1}{x_{1}(t)} \dot{x}_{1}(t)= & r_{1}([t])-a_{11}([t]) x_{1}([t]) \\
& -a_{12}([t]) \sum_{l=0}^{+\infty} K_{12}(-l) x_{2}([t]-l) \\
& -a_{13}([t]) \sum_{l=0}^{+\infty} K_{13}(-l) x_{3}([t]-l), \\
\frac{1}{x_{2}(t)} \dot{x}_{2}(t)= & r_{2}([t])-a_{22}([t]) x_{2}([t]) \\
& -a_{21}([t]) \sum_{l=0}^{+\infty} K_{21}(-l) x_{1}([t]-l) \\
& -a_{23}([t]) \sum_{l=0}^{+\infty} K_{23}(-l) x_{3}([t]-l), \\
\frac{1}{x_{3}(t)} \dot{x}_{3}(t)= & r_{3}([t])-a_{33}([t]) x_{3}([t]) \\
& -a_{31}([t]) \sum_{l=0}^{+\infty} K_{31}(-l) x_{1}([t]-l) \\
\sum_{l=0}^{+\infty} K_{32}(-l) x_{2}([t]-l), & \\
& \\
& \\
&
\end{aligned}
$$

where $[t]$ denotes the integer part of $t, t \in(0,+\infty)$ and $t \neq 0,1,2, \ldots$. Equations of type (5) are known as differential equations with piecewise constant arguments and these equations occupy a position midway between differential 
equations and difference equations. By a solution of (5), we mean a function $\bar{x}=\left(x_{1}, x_{2}, x_{3}\right)^{T}$, which is defined for $t \in$ $[0,+\infty)$ and has the following properties:

(1) $\bar{x}$ is continuous on $[0,+\infty)$;

(2) the derivatives $d x_{1}(t) / d t, d x_{2}(t) / d t, d x_{3}(t) / d t$ exist at each point $t \in[0,+\infty)$ with the possible exception of the points $t \in\{0,1,2, \ldots\}$, where left-sided derivative exists;

(3) the equations in (5) are satisfied on each interval $[k, k+1)$ with $k=0,1,2, \ldots$.

We integrate (5) on any interval of the form $[k, k+1)$, $k=0,1,2, \ldots$, and obtain for $k \leq t<k+1, k=0,1,2, \ldots$

$$
\begin{aligned}
& x_{1}(t)=x_{1}(k) \exp \left\{\left[r_{1}(k)-a_{11}(k) x_{1}(k)\right.\right. \\
& -a_{12}(k) \sum_{l=0}^{+\infty} K_{12}(-l) x_{2}(k-l) \\
& \left.-a_{13}(k) \sum_{l=0}^{+\infty} K_{13}(-l) x_{3}(k-l)\right] \\
& \times(t-k)\}, \\
& x_{2}(t)=x_{2}(k) \exp \left\{\left[r_{2}(k)-a_{22}(k) x_{2}(k)\right.\right. \\
& -a_{21}([t]) \sum_{l=0}^{+\infty} K_{21}(-l) x_{1}(k-l) \\
& \left.-a_{23}(k) \sum_{l=0}^{+\infty} K_{23}(-l) x_{3}(k-l)\right] \\
& \times(t-k)\} \text {, }
\end{aligned}
$$$$
x_{3}(t)=x_{3}(k) \exp \left\{\left[r_{3}(k)-a_{33}(k) x_{3}(k)\right.\right.
$$$$
-a_{31}(k) \sum_{l=0}^{+\infty} K_{31}(-l) x_{1}(k-l)
$$$$
\left.-a_{32}(k) \sum_{l=0}^{+\infty} K_{32}(-l) x_{2}(k-l)\right]
$$$$
\times(t-k)\} \text {. }
$$

Let $t \rightarrow k+1$; then (6) reads as

$$
\begin{aligned}
& x_{1}(k+1)=x_{1}(k) \exp \left\{r_{1}(k)-a_{11}(k) x_{1}(k)\right. \\
& -a_{12}(k) \sum_{l=0}^{+\infty} K_{12}(-l) x_{2}(k-l) \\
& \left.-a_{13}(k) \sum_{l=0}^{+\infty} K_{13}(-l) x_{3}(k-l)\right\}, \\
& x_{2}(k+1)=x_{2}(k) \exp \left\{r_{2}(k)-a_{22}(k) x_{2}(k)\right. \\
& -a_{21}([t]) \sum_{l=0}^{+\infty} K_{21}(-l) x_{1}(k-l) \\
& \left.-a_{23}(k) \sum_{l=0}^{+\infty} K_{23}(-l) x_{3}(k-l)\right\}, \\
& x_{3}(k+1)=x_{3}(k) \exp \left\{r_{3}(k)-a_{33}(k) x_{3}(k)\right. \\
& -a_{31}(k) \sum_{l=0}^{+\infty} K_{31}(-l) x_{1}(k-l) \\
& \left.-a_{32}(k) \sum_{l=0}^{+\infty} K_{32}(-l) x_{2}(k-l)\right\}
\end{aligned}
$$

which is a discrete time analogue of system (3), where $k=$ $0,1,2, \ldots$.

In order to obtain our main results, we assume that the following hold.

(H1) $r_{i}, a_{i j}: Z \rightarrow R^{+}$are positive $\omega$-periodic; that is, $r_{i}(k+\omega)=r_{i}(k)$ and $a_{i j}(k+\omega)=a_{i j}(k)(i, j=1,2,3)$ for any $k \in Z$, where $\omega$, a fixed positive integer, denotes the common period of the parameters in system (7).

(H2) Consider

$$
\begin{aligned}
& 0 \leq \sum_{l=0}^{+\infty} K_{12}(-l)<+\infty, \\
& 0 \leq \sum_{l=0}^{+\infty} K_{13}(-l)<+\infty, \\
& 0 \leq \sum_{l=0}^{+\infty} K_{21}(-l)<+\infty, \\
& 0 \leq \sum_{l=0}^{+\infty} K_{23}(-l)<+\infty,
\end{aligned}
$$




$$
\begin{aligned}
& 0 \leq \sum_{l=0}^{+\infty} K_{31}(-l)<+\infty, \\
& 0 \leq \sum_{l=0}^{+\infty} K_{32}(-l)<+\infty .
\end{aligned}
$$

\section{Existence of Positive Periodic Solutions}

For convenience and simplicity on the following discussion, we always use the notations below throughout the paper:

$$
\begin{gathered}
I_{\omega}:=\{0,1,2, \ldots, \omega-1\}, \\
\bar{f}:=\frac{1}{\omega} \sum_{k=0}^{\omega-1} f(k),
\end{gathered}
$$

where $f(k)$ is an $\omega$-periodic sequence of real numbers defined for $k \in Z$. In order to explore the existence of positive periodic solutions of (7) and for the reader's convenience, we will first summarize below a few concepts and results without proof, borrowing from [21].

Let $X, Y$ be normed vector spaces, $L:$ Dom $L \subset X \rightarrow Y$ a linear mapping, and $N: X \rightarrow Y$ a continuous mapping. The mapping $L$ will be called a Fredholm mapping of index zero if $\operatorname{dim} \operatorname{Ker} L=\operatorname{codim} \operatorname{Im} L<+\infty$ and $\operatorname{Im} L$ is closed in $Y$. If $L$ is a Fredholm mapping of index zero and there exist continuous projectors $P: X \rightarrow X$ and $Q: Y \rightarrow Y$ such that $\operatorname{Im} P=\operatorname{Ker} L, \operatorname{Im} L=\operatorname{Ker} Q=\operatorname{Im}(I-Q)$, it follows that $L \mid \operatorname{Dom} L \cap \operatorname{Ker} P:(I-P) X \rightarrow \operatorname{Im} L$ is invertible. We denote the inverse of that map by $K_{P}$. If $\Omega$ is an open bounded subset of $X$, the mapping $N$ will be called $L$-compact on $\bar{\Omega}$ if $Q N(\bar{\Omega})$ is bounded and $K_{P}(I-Q) N: \bar{\Omega} \rightarrow X$ is compact. Since $\operatorname{Im} Q$ is isomorphic to $\operatorname{Ker} L$, there exist isomorphisms $J: \operatorname{Im} Q \rightarrow \operatorname{Ker} L$.

Lemma 1 (see [21] continuation theorem). Let L be a Fredholm mapping of index zero and let $N$ be L-compact on $\bar{\Omega}$. Suppose

(a) for each $\lambda \in(0,1)$, every solution $x$ of $L x=\lambda N x$ is such that $x \notin \partial \Omega$;

(b) $Q N x \neq 0$ for each $x \in \operatorname{Ker} L \bigcap \partial \Omega$ and $\operatorname{deg}\{J Q N, \Omega \cap \operatorname{Ker} L, 0\} \neq 0$.

Then the equation $L x=N x$ has at least one solution lying in Dom $L \bigcap \bar{\Omega}$.

Lemma 2 (see [17]). Let $g: Z \rightarrow R$ be $\omega$ periodic; that is, $g(k+\omega)=g(k)$; then for any fixed $k_{1}, k_{2} \in I_{\omega}$ and any $k \in Z$, one has

$$
\begin{aligned}
& g(k) \leq g\left(k_{1}\right)+\sum_{s=0}^{\omega-1}|g(s+1)-g(s)|, \\
& g(k) \geq g\left(k_{2}\right)-\sum_{s=0}^{\omega-1}|g(s+1)-g(s)| .
\end{aligned}
$$

Lemma 3. $\left(\widehat{x}_{1}(k), \widehat{x}_{2}(k), \widehat{x}_{3}(k)\right)$ is an $\omega$ periodic solution of (7) with strictly positive components if and only if $\left(\ln \left\{\widehat{x}_{1}(k)\right\}, \ln \left\{\hat{x}_{2}(k)\right\}, \ln \left\{\hat{x}_{3}(k)\right\}\right)$ is an $\omega$ periodic solution of

$$
\begin{aligned}
x_{1}(k+1)-x_{1}(k)= & r_{1}(k)-a_{11}(k) \exp \left(x_{1}(k)\right) \\
& -a_{12}(k) \sum_{l=0}^{+\infty} K_{12}(-l) \exp \left(x_{2}(k-l)\right) \\
& -a_{13}(k) \sum_{l=0}^{+\infty} K_{13}(-l) \exp \left(x_{3}(k-l)\right), \\
x_{2}(k+1)-x_{2}(k)= & r_{2}(k)-a_{22}(k) \exp \left(x_{2}(k)\right) \\
& -a_{21}(k) \sum_{l=0}^{+\infty} K_{21}(-l) \exp \left(x_{1}(k-l)\right) \\
& -a_{23}(k) \sum_{l=0}^{+\infty} K_{23}(-l) \exp \left(x_{3}(k-l)\right), \\
x_{3}(k+1)-x_{3}(k)= & r_{3}(k)-a_{33}(k) \exp \left(x_{3}(k)\right) \\
& -a_{31}(k) \sum_{l=0}^{+\infty} K_{31}(-l) \exp \left(x_{1}(k-l)\right) \\
& -a_{32}(k) \sum_{l=0}^{+\infty} K_{32}(-l) \exp \left(x_{2}(k-l)\right) .
\end{aligned}
$$

The proofs of Lemma 3 are trivial, so we omitted the details here.

Define

$$
l_{3}=\left\{z=\{z(k)\}: z(k) \in R^{3}, k \in Z\right\}
$$

For $a=\left(a_{1}, a_{2}, a_{3}\right)^{T} \in R^{3}$, define $|a|=\max \left\{\left|a_{1}\right|,\left|a_{2}\right|,\left|a_{3}\right|\right\}$. Let $l^{\omega} \subset l_{3}$ denote the subspace of all $\omega$ periodic sequences equipped with the usual supremum norm $\|\cdot\|$; that is, $\|z\|=$ $\max _{k \in I_{\omega}}|z(k)|$, for any $z=\{z(k): k \in Z\} \in l^{\omega}$. It is easy to show that $l_{\omega}$ is a finite-dimensional Banach space.

Let

$$
\begin{gathered}
l_{0}^{\omega}=\left\{z=\{z(k)\} \in l^{\omega}: \sum_{k=0}^{\omega-1} z(k)=0\right\}, \\
l_{c}^{\omega}=\left\{z=\{z(k)\} \in l^{\omega}: z(k)=h \in R^{3}, k \in Z\right\},
\end{gathered}
$$

then it follows that $l_{0}^{\omega}$ and $l_{c}^{\omega}$ are both closed linear subspaces of $l^{\omega}$ and

$$
l^{\omega}=l_{0}^{\omega}+l_{c}^{\omega}, \quad \operatorname{dim} l_{c}^{\omega}=3 .
$$

Next, we will be ready to establish our result. 
Theorem 4. Let $B_{3}, B_{6}, B_{11}, B_{15}, B_{17}, B_{22}, B_{33}, B_{35}, B_{39}$, and $B_{41}$ be defined by (33), (37), (49), (57), (61), (73), (81), (86), (94), and (98), respectively, and set

$$
\begin{aligned}
\theta_{1}= & \bar{a}_{11} \exp \left(B_{3}\right)+\bar{a}_{12} \sum_{l=1}^{\infty} K_{12}(-l) \exp \left(B_{6}\right), \\
\theta_{2}= & \bar{a}_{11} \exp \left(B_{3}\right)+\bar{a}_{13} \sum_{l=1}^{\infty} K_{13}(-l) \exp \left(B_{11}\right) \\
\theta_{3}= & \bar{a}_{11} \exp \left(B_{17}\right)+\bar{a}_{12} \sum_{l=1}^{\infty} K_{12}(-l) \exp \left(B_{15}\right), \\
\theta_{4}= & \bar{a}_{12} \sum_{l=1}^{\infty} K_{12}(-l) \exp \left(B_{15}\right) \\
& +\bar{a}_{13} \sum_{l=1}^{\infty} K_{13}(-l) \exp \left(B_{22}\right), \\
\theta_{5}= & \bar{a}_{11} \exp \left(B_{35}\right)+\bar{a}_{13} \sum_{l=1}^{\infty} K_{13}(-l) \exp \left(B_{33}\right), \\
\theta_{6}= & \bar{a}_{13} \sum_{l=1}^{\infty} K_{13}(-l) \exp \left(B_{39}\right), \\
\theta_{7}= & \bar{a}_{12} \sum_{l=1}^{\infty} K_{12}(-l) \exp \left(B_{39}\right) \\
& +\bar{a}_{13} \sum_{l=1}^{\infty} K_{13}(-l) \exp \left(B_{41}\right)
\end{aligned}
$$

Suppose that (H1), (H2) and (H3) $\bar{r}_{1}>\left\{\theta_{1}, \theta_{2}, \theta_{3}, \theta_{4}, \theta_{5}\right.$, $\left.\theta_{6}, \theta_{7}\right\}$ hold, then system (7) has at least an $\omega$ periodic solution with positive components.

Proof. Let $X=Y=l^{\omega}$,

$$
\begin{gathered}
(L z)(k)=z(k+1)-z(k), \\
(N z)(k)=\left(\begin{array}{l}
f_{1}(k) \\
f_{2}(k) \\
f_{3}(k)
\end{array}\right),
\end{gathered}
$$

where $z \in X, k \in Z$, and

$$
\begin{aligned}
f_{1}(k)= & r_{1}(k)-a_{11}(k) \exp \left(x_{1}(k)\right) \\
& -a_{12}(k) \sum_{l=0}^{+\infty} K_{12}(-l) \exp \left(x_{2}(k-l)\right) \\
& -a_{13}(k) \sum_{l=0}^{+\infty} K_{13}(-l) \exp \left(x_{3}(k-l)\right),
\end{aligned}
$$

$$
\begin{aligned}
f_{2}(k)= & r_{2}(k)-a_{22}(k) \exp \left(x_{2}(k)\right) \\
& -a_{21}(k) \sum_{l=0}^{+\infty} K_{21}(-l) \exp \left(x_{1}(k-l)\right) \\
& -a_{23}(k) \sum_{l=0}^{+\infty} K_{23}(-l) \exp \left(x_{3}(k-l)\right), \\
f_{3}(k)= & r_{3}(k)-a_{33}(k) \exp \left(x_{3}(k)\right) \\
& -a_{31}(k) \sum_{l=0}^{+\infty} K_{31}(-l) \exp \left(x_{1}(k-l)\right) \\
& -a_{32}(k) \sum_{l=0}^{+\infty} K_{32}(-l) \exp \left(x_{2}(k-l)\right) .
\end{aligned}
$$

Then it is trivial to see that $L$ is a bounded linear operator and

$$
\begin{gathered}
\operatorname{Ker} L=l_{c}^{\omega}, \quad \operatorname{Im} L=l_{0}^{\omega}, \\
\operatorname{dim} \operatorname{Ker} L=3=\operatorname{codim} \operatorname{Im} L,
\end{gathered}
$$

then it follows that $L$ is a Fredholm mapping of index zero. Define

$$
\begin{aligned}
& P y=\frac{1}{\omega} \sum_{s=0}^{\omega-1} y(s), \quad y \in X, \\
& \mathrm{Q} z=\frac{1}{\omega} \sum_{s=0}^{\omega-1} z(s), \quad z \in Y .
\end{aligned}
$$

It is not difficult to show that $P$ and $Q$ are continuous projectors such that

$$
\operatorname{Im} P=\operatorname{Ker} L, \quad \operatorname{Im} L=\operatorname{Ker} Q=\operatorname{Im}(I-Q) .
$$

Furthermore, the generalized inverse (to $L$ ) $K_{P}: \operatorname{Im} L \rightarrow$ $\operatorname{Ker} P \bigcap \operatorname{Dom} L$ exists and is given by

$$
K_{P}(z)=\sum_{s=0}^{\omega-1} z(s)-\frac{1}{\omega} \sum_{s=0}^{\omega-1}(\omega-s) z(s)
$$

Obviously, $Q N$ and $K_{P}(I-Q) N$ are continuous. Since $X$ is a finite-dimensional Banach space, it is not difficult to show that $\overline{K_{P}(I-Q) N(\bar{\Omega})}$ is compact for any open bounded set $\Omega \subset X$. Moreover, $Q N(\bar{\Omega})$ is bounded. Thus, $N$ is $L$-compact on $\bar{\Omega}$ with any open bounded set $\Omega \subset X$.

Now we are at the point to search for an appropriate open, bounded subset $\Omega$ for the application of the continuation 
theorem. Corresponding to the operator equation $L z=\lambda \mathrm{Nz}$, $\lambda \in(0,1)$, we have

$$
\begin{aligned}
x_{1}(k+1)-x_{1}(k)=\lambda[ & r_{1}(k)-a_{11}(k) \exp \left(x_{1}(k)\right) \\
& -a_{12}(k) \sum_{l=0}^{+\infty} K_{12}(-l) \exp \left(x_{2}(k-l)\right) \\
& \left.-a_{13}(k) \sum_{l=0}^{+\infty} K_{13}(-l) \exp \left(x_{3}(k-l)\right)\right],
\end{aligned}
$$$$
x_{2}(k+1)-x_{2}(k)=\lambda\left[r_{2}(k)-a_{22}(k) \exp \left(x_{2}(k)\right)\right.
$$$$
-a_{21}(k) \sum_{l=0}^{+\infty} K_{21}(-l) \exp \left(x_{1}(k-l)\right)
$$$$
\left.-a_{23}(k) \sum_{l=0}^{+\infty} K_{23}(-l) \exp \left(x_{3}(k-l)\right)\right],
$$$$
x_{3}(k+1)-x_{3}(k)=\lambda\left[r_{3}(k)-a_{33}(k) \exp \left(x_{3}(k)\right)\right.
$$$$
\begin{aligned}
& -a_{31}(k) \sum_{l=0}^{+\infty} K_{31}(-l) \exp \left(x_{1}(k-l)\right) \\
& \left.-a_{32}(k) \sum_{l=0}^{+\infty} K_{32}(-l) \exp \left(x_{2}(k-l)\right)\right] .
\end{aligned}
$$

Suppose that $z(k)=\left(x_{1}(k), x_{2}(k), x_{3}(k)\right)^{T} \in X$ is an arbitrary solution of system (22) for a certain $\lambda \in(0,1)$; summing both sides of (22) from 0 to $\omega-1$ with respect to $k$, respectively, we obtain

$$
\begin{gathered}
\sum_{k=0}^{\omega-1}\left[a_{11}(k) \exp \left(x_{1}(k)\right)+a_{12}(k) \sum_{l=0}^{+\infty} K_{12}(-l) \exp \left(x_{2}(k-l)\right)\right. \\
\left.+a_{13}(k) \sum_{l=0}^{+\infty} K_{13}(-l) \exp \left(x_{3}(k-l)\right)\right]=\bar{r}_{1} \omega,
\end{gathered}
$$$$
\sum_{k=0}^{\omega-1}\left[a_{22}(k) \exp \left(x_{2}(k)\right)+a_{21}(k) \sum_{l=0}^{+\infty} K_{21}(-l) \exp \left(x_{1}(k-l)\right)\right.
$$$$
\left.+a_{23}(k) \sum_{l=0}^{+\infty} K_{23}(-l) \exp \left(x_{3}(k-l)\right)\right]=\bar{r}_{2} \omega,
$$

$$
\begin{gathered}
\sum_{k=0}^{\omega-1}\left[a_{33}(k) \exp \left(x_{3}(k)\right)+a_{31}(k) \sum_{l=0}^{+\infty} K_{31}(-l) \exp \left(x_{1}(k-l)\right)\right. \\
\left.+a_{32}(k) \sum_{l=0}^{+\infty} K_{32}(-l) \exp \left(x_{2}(k-l)\right)\right]=\bar{r}_{3} \omega .
\end{gathered}
$$

It follows from (22), (23), (24), and (25) that

$$
\begin{aligned}
& \sum_{k=0}^{\omega-1}\left|x_{1}(k+1)-x_{1}(k)\right| \leq 2 \bar{r}_{1} \omega, \\
& \sum_{k=0}^{\omega-1}\left|x_{2}(k+1)-x_{2}(k)\right| \leq 2 \bar{r}_{2} \omega, \\
& \sum_{k=0}^{\omega-1}\left|x_{3}(k+1)-x_{3}(k)\right| \leq 2 \bar{r}_{3} \omega .
\end{aligned}
$$

In view of the hypothesis that $z=\{z(k)\} \in X$, there exist $\xi_{i}, \eta_{i} \in I_{\omega}$ such that

$$
\begin{aligned}
& x_{i}\left(\xi_{i}\right)=\min _{k \in I_{\omega}}\left\{x_{i}(k)\right\}, \\
& x_{i}\left(\eta_{i}\right)=\max _{k \in I_{\omega}}\left\{x_{i}(k)\right\} \quad(i=1,2,3) .
\end{aligned}
$$

By (23), (24), and (25), we have

$$
\begin{aligned}
& \bar{a}_{11}(k) \exp \left(x_{1}\left(\xi_{1}\right)\right) \leq \sum_{k=0}^{\omega-1} a_{11}(k) \exp \left(x_{1}(k)\right)<\bar{r}_{1} \omega, \\
& \bar{a}_{22}(k) \exp \left(x_{2}\left(\xi_{2}\right)\right) \leq \sum_{k=0}^{\omega-1} a_{22}(k) \exp \left(x_{2}(k)\right)<\bar{r}_{2} \omega, \\
& \bar{a}_{33}(k) \exp \left(x_{3}\left(\xi_{3}\right)\right) \leq \sum_{k=0}^{\omega-1} a_{33}(k) \exp \left(x_{3}(k)\right)<\bar{r}_{3} \omega .
\end{aligned}
$$

Thus

$$
\begin{aligned}
& x_{1}\left(\xi_{1}\right)<\ln \left[\frac{\bar{r}_{1}}{\bar{a}_{11}}\right], \\
& x_{2}\left(\xi_{2}\right)<\ln \left[\frac{\bar{r}_{2}}{\bar{a}_{22}}\right], \\
& x_{3}\left(\xi_{3}\right)<\ln \left[\frac{\bar{r}_{3}}{\bar{a}_{33}}\right] .
\end{aligned}
$$

In the sequel, we consider six cases.

(a) If $x_{1}\left(\eta_{1}\right) \geq x_{2}\left(\eta_{2}\right) \geq x_{3}\left(\eta_{3}\right)$, then it follows from (23) that

$$
\begin{aligned}
& {\left[\bar{a}_{11}+\bar{a}_{12} \sum_{l=0}^{+\infty} K_{12}(-l)+\bar{a}_{13} \sum_{l=0}^{+\infty} K_{13}(-l)\right]} \\
& \quad \times \exp \left(x_{1}\left(\eta_{1}\right)\right) \omega \geq \bar{r}_{1} \omega
\end{aligned}
$$


which leads to

$$
\begin{aligned}
& x_{1}\left(\eta_{1}\right) \\
& \quad>\ln \left[\frac{\bar{r}_{1}}{\bar{a}_{11}+\bar{a}_{12} \sum_{l=0}^{+\infty} K_{12}(-l)+\bar{a}_{13} \sum_{l=0}^{+\infty} K_{13}(-l)}\right]:=M_{1},
\end{aligned}
$$

It follows from (29), (31), and Lemma 2 that

$$
\begin{aligned}
x_{1}(k) & \leq x_{1}\left(\xi_{1}\right)+\sum_{s=0}^{\omega-1}\left|x_{1}(s+1)-x_{1}(s)\right| \\
& \leq \ln \left[\frac{\bar{r}_{1}}{\bar{a}_{11}}\right]+2 \bar{r}_{1} \omega:=B_{1}, \\
x_{1}(k) & \geq x_{1}\left(\eta_{1}\right)-\sum_{s=0}^{\omega-1}\left|x_{1}(s+1)-x_{1}(s)\right| \\
& \geq M_{1}-2 \bar{r}_{1} \omega:=B_{2} .
\end{aligned}
$$

By (32), we derive

$$
\max _{k \in I_{\omega}}\left\{x_{1}(k)\right\} \leq \max \left\{\left|B_{1}\right|,\left|B_{2}\right|\right\}:=B_{3} .
$$

From (23) and (33), we obtain that

$$
\begin{aligned}
& \bar{a}_{11} \omega \exp \left(B_{3}\right)+\left[\bar{a}_{12} \sum_{l=0}^{+\infty} K_{12}(-l)+\bar{a}_{13} \sum_{l=0}^{+\infty} K_{13}(-l)\right] \\
& \times \omega \exp \left(x_{2}\left(\eta_{2}\right)\right) \geq \bar{r}_{1} \omega .
\end{aligned}
$$

Then

$$
x_{2}\left(\eta_{2}\right) \geq \ln \left[\frac{\bar{r}_{1}-\bar{a}_{11} \exp \left(B_{3}\right)}{\bar{a}_{12} \sum_{l=0}^{+\infty} K_{12}(-l)+\bar{a}_{13} \sum_{l=0}^{+\infty} K_{13}(-l)}\right] .
$$

Thus by (29), (35), and Lemma 2, we get

$$
\begin{aligned}
x_{2}(k) \leq & x_{2}\left(\xi_{2}\right)+\sum_{s=0}^{\omega-1}\left|x_{2}(s+1)-x_{2}(s)\right| \\
\leq & \ln \left[\frac{\bar{r}_{2}}{\bar{a}_{22}}\right]+2 \bar{r}_{2} \omega:=B_{4}, \\
x_{2}(k) \geq & x_{2}\left(\eta_{2}\right)-\sum_{s=0}^{\omega-1}\left|x_{2}(s+1)-x_{2}(s)\right| \\
\geq & \ln \left[\frac{\bar{r}_{1}-\bar{a}_{11} \exp \left(B_{3}\right)}{\bar{a}_{12} \sum_{l=0}^{+\infty} K_{12}(-l)+\bar{a}_{13} \sum_{l=0}^{+\infty} K_{13}(-l)}\right] \\
& -2 \bar{r}_{2} \omega:=B_{5} .
\end{aligned}
$$

It follows from (36) that

$$
\max _{k \in I_{\omega}}\left\{x_{2}(k)\right\} \leq \max \left\{\left|B_{4}\right|,\left|B_{5}\right|\right\}:=B_{6} .
$$

In view of (33), (37), and (23), we get

$$
\begin{aligned}
& \bar{a}_{11} \omega \exp \left(B_{3}\right)+\bar{a}_{12} \omega \sum_{l=0}^{+\infty} K_{12}(-l) \exp \left(B_{6}\right) \\
& +\bar{a}_{13} \omega \sum_{l=0}^{+\infty} K_{13}(-l) \exp \left(x_{3}\left(\eta_{3}\right)\right) \geq \bar{r}_{1} \omega .
\end{aligned}
$$

Then

$$
\begin{aligned}
& x_{3}\left(\eta_{3}\right) \\
& \quad \geq \ln \left[\frac{\bar{r}_{1}-\bar{a}_{11} \exp \left(B_{3}\right)-\bar{a}_{12} \sum_{l=0}^{+\infty} K_{12}(-l) \exp \left(B_{6}\right)}{\bar{a}_{13} \sum_{l=0}^{+\infty} K_{13}(-l)}\right] .
\end{aligned}
$$

Thus by (29), (39) and Lemma 2, we get

$$
\begin{aligned}
x_{3}(k) & \leq x_{3}\left(\xi_{3}\right)+\sum_{s=0}^{\omega-1}\left|x_{3}(s+1)-x_{3}(s)\right| \\
& \leq \ln \left[\frac{\bar{r}_{3}}{\bar{a}_{33}}\right]+2 \bar{r}_{3} \omega:=B_{7},
\end{aligned}
$$

$$
\begin{aligned}
x_{3}(k) \geq & x_{3}\left(\eta_{3}\right)-\sum_{s=0}^{\omega-1}\left|x_{3}(s+1)-x_{3}(s)\right| \\
\geq & \ln \left[\frac{\bar{r}_{1}-\bar{a}_{11} \exp \left(B_{3}\right)-\bar{a}_{12} \sum_{l=0}^{+\infty} K_{12}(-l) \exp \left(B_{6}\right)}{\bar{a}_{13} \sum_{l=0}^{+\infty} K_{13}(-l)}\right] \\
& -2 \bar{r}_{3} \omega:=B_{8} .
\end{aligned}
$$

It follows from (40) that

$$
\max _{k \in I_{\omega}}\left\{x_{3}(k)\right\} \leq \max \left\{\left|B_{7}\right|,\left|B_{8}\right|\right\}:=B_{9}
$$

(b) If $x_{1}\left(\eta_{1}\right) \geq x_{3}\left(\eta_{3}\right) \geq x_{2}\left(\eta_{2}\right)$, then it follows from (23) that

$$
\begin{aligned}
& {\left[\bar{a}_{11}+\bar{a}_{12} \sum_{l=0}^{+\infty} K_{12}(-l)+\bar{a}_{13} \sum_{l=0}^{+\infty} K_{13}(-l)\right]} \\
& \quad \times \exp \left(x_{1}\left(\eta_{1}\right)\right) \omega \geq \bar{r}_{1} \omega
\end{aligned}
$$

which leads to

$$
\begin{aligned}
& x_{1}\left(\eta_{1}\right) \\
& >\ln \left[\frac{\bar{r}_{1}}{\bar{a}_{11}+\bar{a}_{12} \sum_{l=0}^{+\infty} K_{12}(-l)+\bar{a}_{13} \sum_{l=0}^{+\infty} K_{13}(-l)}\right]:=M_{1} .
\end{aligned}
$$


It follows from (29), (43), and Lemma 2 that

$$
\begin{aligned}
x_{1}(k) & \leq x_{1}\left(\xi_{1}\right)+\sum_{s=0}^{\omega-1}\left|x_{1}(s+1)-x_{1}(s)\right| \\
& \leq \ln \left[\frac{\bar{r}_{1}}{\bar{a}_{11}}\right]+2 \bar{r}_{1} \omega:=B_{1}, \\
x_{1}(k) & \geq x_{1}\left(\eta_{1}\right)-\sum_{s=0}^{\omega-1}\left|x_{1}(s+1)-x_{1}(s)\right| \\
& \geq M_{1}-2 \bar{r}_{1} \omega:=B_{2} .
\end{aligned}
$$

By (44), we derive

$$
\max _{k \in I_{\omega}}\left\{x_{1}(k)\right\}<\max \left\{\left|B_{1}\right|,\left|B_{2}\right|\right\}:=B_{3} .
$$

From (23) and (45), we obtain that

$$
\begin{aligned}
& \bar{a}_{11} \omega \exp \left(B_{3}\right) \\
& +\left[\bar{a}_{12} \sum_{l=0}^{+\infty} K_{12}(-l)+\bar{a}_{13} \sum_{l=0}^{+\infty} K_{13}(-l)\right] \\
& \quad \times \omega \exp \left(x_{3}\left(\eta_{3}\right)\right) \geq \bar{r}_{1} \omega .
\end{aligned}
$$

Then

$$
x_{3}\left(\eta_{3}\right) \geq \ln \left[\frac{\bar{r}_{1}-\bar{a}_{11} \exp \left(B_{3}\right)}{\bar{a}_{12} \sum_{l=0}^{+\infty} K_{12}(-l)+\bar{a}_{13} \sum_{l=0}^{+\infty} K_{13}(-l)}\right],
$$

Thus by (29), (47), and Lemma 2, we get

$$
\begin{aligned}
x_{3}(k) \leq & x_{3}\left(\xi_{3}\right)+\sum_{s=0}^{\omega-1}\left|x_{3}(s+1)-x_{3}(s)\right| \\
\leq & \ln \left[\frac{\bar{r}_{3}}{\bar{a}_{33}}\right]+2 \bar{r}_{3} \omega:=B_{7}, \\
x_{3}(k) \geq & x_{3}\left(\eta_{3}\right)-\sum_{s=0}^{\omega-1}\left|x_{3}(s+1)-x_{3}(s)\right| \\
\geq & \ln \left[\frac{\bar{r}_{1}-\bar{a}_{11} \exp \left(B_{3}\right)}{\bar{a}_{12} \sum_{l=0}^{+\infty} K_{12}(-l)+\bar{a}_{13} \sum_{l=0}^{+\infty} K_{13}(-l)}\right] \\
& -2 \bar{r}_{3} \omega:=B_{10} .
\end{aligned}
$$

It follows from (48) that

$$
\max _{k \in I_{\omega}}\left\{x_{3}(k)\right\} \leq \max \left\{\left|B_{7}\right|,\left|B_{10}\right|\right\}:=B_{11} .
$$

In view of (45), (49), and (23), we get

$$
\begin{gathered}
\bar{a}_{11} \omega \exp \left(B_{3}\right)+\bar{a}_{12} \omega \sum_{l=0}^{+\infty} K_{12}(-l) \exp \left(x_{2}\left(\eta_{2}\right)\right) \\
+\bar{a}_{13} \omega \sum_{l=0}^{+\infty} K_{13}(-l) \exp \left(B_{11}\right) \geq \bar{r}_{1} \omega .
\end{gathered}
$$

Then

$$
\begin{aligned}
& x_{2}\left(\eta_{2}\right) \\
& \qquad \geq \ln \left[\frac{\bar{r}_{1}-\bar{a}_{11} \exp \left(B_{3}\right)-\bar{a}_{13} \sum_{l=0}^{+\infty} K_{13}(-l) \exp \left(B_{11}\right)}{\bar{a}_{12} \sum_{l=0}^{+\infty} K_{12}(-l)}\right] .
\end{aligned}
$$

Thus by (29), (51), and Lemma 2, we get

$$
\begin{aligned}
x_{3}(k) \leq & x_{3}\left(\xi_{3}\right)+\sum_{s=0}^{\omega-1}\left|x_{3}(s+1)-x_{3}(s)\right| \\
\leq & \ln \left[\frac{\bar{r}_{3}}{\bar{a}_{33}}\right]+2 \bar{r}_{3} \omega:=B_{7}, \\
x_{3}(k) \geq & x_{3}\left(\eta_{3}\right)-\sum_{s=0}^{\omega-1}\left|x_{3}(s+1)-x_{3}(s)\right| \\
\geq & \ln \left[\frac{\bar{r}_{1}-\bar{a}_{11} \exp \left(B_{3}\right)-\bar{a}_{13} \sum_{l=0}^{+\infty} K_{13}(-l) \exp \left(B_{11}\right)}{\bar{a}_{12} \sum_{l=0}^{+\infty} K_{12}(-l)}\right] \\
& -2 \bar{r}_{3} \omega:=B_{12} .
\end{aligned}
$$

It follows from (52) that

$$
\max _{k \in I_{\omega}}\left\{x_{3}(k)\right\} \leq \max \left\{\left|B_{7}\right|,\left|B_{12}\right|\right\}:=B_{13} .
$$

(c) If $x_{2}\left(\eta_{2}\right) \geq x_{1}\left(\eta_{1}\right) \geq x_{3}\left(\eta_{3}\right)$, then it follows from (23) that

$$
\begin{aligned}
& {\left[\bar{a}_{11}+\bar{a}_{12} \sum_{l=0}^{+\infty} K_{12}(-l)+\bar{a}_{13} \sum_{l=0}^{+\infty} K_{13}(-l)\right]} \\
& \quad \times \exp \left(x_{2}\left(\eta_{2}\right)\right) \omega \geq \bar{r}_{1} \omega
\end{aligned}
$$

which leads to

$$
\begin{aligned}
& x_{2}\left(\eta_{2}\right) \\
& \quad>\ln \left[\frac{\bar{r}_{1}}{\bar{a}_{11}+\bar{a}_{12} \sum_{l=0}^{+\infty} K_{12}(-l)+\bar{a}_{13} \sum_{l=0}^{+\infty} K_{13}(-l)}\right]:=M_{1} .
\end{aligned}
$$

It follows from (29), (55), and Lemma 2 that

$$
\begin{aligned}
x_{2}(k) & \leq x_{2}\left(\xi_{2}\right)+\sum_{s=0}^{\omega-1}\left|x_{2}(s+1)-x_{2}(s)\right| \\
& \leq \ln \left[\frac{\bar{r}_{2}}{\bar{a}_{22}}\right]+2 \bar{r}_{2} \omega:=B_{4}, \\
x_{2}(k) & \geq x_{2}\left(\eta_{2}\right)-\sum_{s=0}^{\omega-1}\left|x_{2}(s+1)-x_{2}(s)\right| \\
& \geq M_{1}-2 \bar{r}_{2} \omega:=B_{14} .
\end{aligned}
$$

By (56), we derive

$$
\max _{k \in I_{\omega}}\left\{x_{2}(k)\right\} \leq \max \left\{\left|B_{4}\right|,\left|B_{14}\right|\right\}:=B_{15} .
$$


From (23) and (57), we obtain that

$$
\begin{gathered}
{\left[\bar{a}_{11}+\bar{a}_{13} \sum_{l=0}^{+\infty} K_{13}(-l)\right] \omega \exp \left(x_{1}\left(\eta_{1}\right)\right)} \\
+\left[\bar{a}_{12} \sum_{l=0}^{+\infty} \mathrm{K}_{12}(-l)\right] \omega \exp \left(B_{15}\right) \geq \bar{r}_{1} \omega .
\end{gathered}
$$

Then

$$
x_{1}\left(\eta_{1}\right) \geq \ln \left[\frac{\bar{r}_{1}-\bar{a}_{12} \sum_{l=0}^{+\infty} K_{12}(-l) \exp \left(B_{15}\right)}{\bar{a}_{11}+\bar{a}_{13} \sum_{l=0}^{+\infty} K_{13}(-l)}\right] .
$$

Thus by (29), (59), and Lemma 2, we get

$$
\begin{aligned}
x_{1}(k) & \leq x_{1}\left(\xi_{1}\right)+\sum_{s=0}^{\omega-1}\left|x_{1}(s+1)-x_{1}(s)\right| \\
& \leq \ln \left[\frac{\bar{r}_{1}}{\bar{a}_{11}}\right]+2 \bar{r}_{1} \omega:=B_{1}, \\
x_{1}(k) & \geq x_{1}\left(\eta_{1}\right)-\sum_{s=0}^{\omega-1}\left|x_{1}(s+1)-x_{1}(s)\right| \\
& \geq \ln \left[\frac{\bar{r}_{1}-\bar{a}_{12} \sum_{l=0}^{+\infty} K_{12}(-l) \exp \left(B_{15}\right)}{\bar{a}_{11}+\bar{a}_{13} \sum_{l=0}^{+\infty} K_{13}(-l)}\right]-2 \bar{r}_{1} \omega:=B_{16} .
\end{aligned}
$$

It follows from (60) that

$$
\max _{k \in I_{\omega}}\left\{x_{1}(k)\right\} \leq \max \left\{\left|B_{1}\right|,\left|B_{16}\right|\right\}:=B_{17} .
$$

In view of (57), (61), and (23), we get

$$
\begin{gathered}
\bar{a}_{11} \omega \exp \left(B_{17}\right)+\bar{a}_{12} \omega \sum_{l=0}^{+\infty} K_{12}(-l) \exp \left(B_{15}\right) \\
+\bar{a}_{13} \omega \sum_{l=0}^{+\infty} K_{13}(-l) \exp \left(x_{3}\left(\eta_{3}\right)\right) \geq \bar{r}_{1} \omega .
\end{gathered}
$$

Then

$$
\begin{aligned}
& x_{3}\left(\eta_{3}\right) \\
& \quad \geq \ln \left[\frac{\bar{r}_{1}-\bar{a}_{11} \exp \left(B_{17}\right)-\bar{a}_{12} \sum_{l=0}^{+\infty} K_{12}(-l) \exp \left(B_{15}\right)}{\bar{a}_{13} \sum_{l=0}^{+\infty} K_{13}(-l)}\right] .
\end{aligned}
$$

Thus by (29), (63), and Lemma 2, we get

$$
\begin{aligned}
x_{3}(k) & \leq x_{3}\left(\xi_{3}\right)+\sum_{s=0}^{\omega-1}\left|x_{3}(s+1)-x_{3}(s)\right| \\
& \leq \ln \left[\frac{2 \bar{r}_{3}}{\bar{a}_{33}}\right]+2 \bar{r}_{3} \omega:=B_{7}, \\
x_{3}(k) & \geq y\left(\eta_{3}\right)-\sum_{s=0}^{\omega-1}\left|x_{3}(s+1)-x_{3}(s)\right|
\end{aligned}
$$

$$
\begin{aligned}
& \geq \ln \left[\frac{\bar{r}_{1}-\bar{a}_{11} \exp \left(B_{17}\right)}{\bar{a}_{13} \sum_{l=0}^{+\infty} K_{13}(-l)}\right. \\
& \left.\quad-\frac{\bar{a}_{12} \sum_{l=0}^{+\infty} K_{12}(-l) \exp \left(B_{15}\right)}{\bar{a}_{13} \sum_{l=0}^{+\infty} K_{13}(-l)}\right] \\
& \quad-2 \bar{r}_{3} \omega:=B_{18} .
\end{aligned}
$$

It follows from (64) that

$$
\max _{k \in I_{\omega}}\left\{x_{3}(k)\right\} \leq \max \left\{\left|B_{7}\right|,\left|B_{18}\right|\right\}:=B_{19} .
$$
that

(d) If $x_{2}\left(\eta_{2}\right) \geq x_{3}\left(\eta_{3}\right) \geq x_{1}\left(\eta_{1}\right)$, then it follows from (23)

$$
\begin{aligned}
& {\left[\bar{a}_{11}+\bar{a}_{12} \sum_{l=0}^{+\infty} K_{12}(-l)+\bar{a}_{13} \sum_{l=0}^{+\infty} K_{13}(-l)\right]} \\
& \quad \times \exp \left(x_{2}\left(\eta_{2}\right)\right) \omega \geq \bar{r}_{1} \omega
\end{aligned}
$$

which leads to

$x_{2}\left(\eta_{2}\right)$

$$
>\ln \left[\frac{\bar{r}_{1}}{\bar{a}_{11}+\bar{a}_{12} \sum_{l=0}^{+\infty} K_{12}(-l)+\bar{a}_{13} \sum_{l=0}^{+\infty} K_{13}(-l)}\right]:=M_{1} .
$$

It follows from (29), (67), and Lemma 2 that

$$
\begin{aligned}
x_{2}(k) & \leq x_{2}\left(\xi_{2}\right)+\sum_{s=0}^{\omega-1}\left|x_{2}(s+1)-x_{2}(s)\right| \\
& \leq \ln \left[\frac{\bar{r}_{2}}{\bar{a}_{22}}\right]+2 \bar{r}_{2} \omega:=B_{4}, \\
x_{2}(k) & \geq x_{2}\left(\eta_{2}\right)-\sum_{s=0}^{\omega-1}\left|x_{2}(s+1)-x_{2}(s)\right| \\
& \geq M_{1}-2 \bar{r}_{2} \omega:=B_{14} .
\end{aligned}
$$

By (56), we derive

$$
\max _{k \in I_{\omega}}\left\{x_{2}(k)\right\} \leq \max \left\{\left|B_{4}\right|,\left|B_{14}\right|\right\}:=B_{20} .
$$

From (23) and (57), we obtain that

$$
\begin{aligned}
& {\left[\bar{a}_{11}+\bar{a}_{13} \sum_{l=0}^{+\infty} K_{13}(-l)\right] \omega \exp \left(x_{3}\left(\eta_{3}\right)\right)} \\
& +\left[\bar{a}_{12} \sum_{l=0}^{+\infty} K_{12}(-l)\right] \omega \exp \left(B_{20}\right) \geq \bar{r}_{1} \omega .
\end{aligned}
$$


Then

$$
x_{3}\left(\eta_{3}\right) \geq \ln \left[\frac{\bar{r}_{1}-\bar{a}_{12} \sum_{l=0}^{+\infty} K_{12}(-l) \exp \left(B_{20}\right)}{\bar{a}_{11}+\bar{a}_{13} \sum_{l=0}^{+\infty} K_{13}(-l)}\right] .
$$

Thus by (29), (71), and Lemma 2, we get

$$
\begin{aligned}
x_{3}(k) & \leq x_{3}\left(\xi_{3}\right)+\sum_{s=0}^{\omega-1}\left|x_{3}(s+1)-x_{3}(s)\right| \\
& \leq \ln \left[\frac{\bar{r}_{3}}{\bar{a}_{33}}\right]+2 \bar{r}_{3} \omega:=B_{7}, \\
x_{3}(k) & \geq x_{3}\left(\eta_{3}\right)-\sum_{s=0}^{\omega-1}\left|x_{3}(s+1)-x_{3}(s)\right| \\
& \geq \ln \left[\frac{\bar{r}_{1}-\bar{a}_{12} \sum_{l=0}^{+\infty} K_{12}(-l) \exp \left(B_{20}\right)}{\bar{a}_{11}+\bar{a}_{13} \sum_{l=0}^{+\infty} K_{13}(-l)}\right]-2 \bar{r}_{3} \omega:=B_{21} .
\end{aligned}
$$

It follows from (72) that

$$
\max _{k \in I_{\omega}}\left\{x_{3}(k)\right\} \leq \max \left\{\left|B_{7}\right|,\left|B_{20}\right|\right\}:=B_{22} .
$$

In view of (69), (73), and (23), we get

$$
\begin{gathered}
\bar{a}_{11} \omega \exp \left(x_{1}\left(\eta_{1}\right)\right)+\bar{a}_{12} \omega \sum_{l=0}^{+\infty} K_{12}(-l) \exp \left(B_{20}\right) \\
+\bar{a}_{13} \omega \sum_{l=0}^{+\infty} K_{13}(-l) \exp \left(B_{22}\right) \geq \bar{r}_{1} \omega .
\end{gathered}
$$

Then

$$
\begin{aligned}
& x_{1}\left(\eta_{1}\right) \\
& \geq \ln \left[\frac{\bar{r}_{1}-\bar{a}_{12} \sum_{l=0}^{+\infty} K_{12}(-l) \exp \left(B_{20}\right)}{\bar{a}_{13} \sum_{l=0}^{+\infty} K_{13}(-l)}\right. \\
& \\
&\left.\quad-\frac{\bar{a}_{13} \sum_{l=0}^{+\infty} K_{13}(-l) \exp \left(B_{22}\right)}{\bar{a}_{13} \sum_{l=0}^{+\infty} K_{13}(-l)}\right] .
\end{aligned}
$$

Thus by (29), (75), and Lemma 2, we get

$$
\begin{aligned}
& x_{1}(k) \leq x_{1}\left(\xi_{1}\right)+\sum_{s=0}^{\omega-1}\left|x_{1}(s+1)-x_{1}(s)\right| \\
& \leq \ln \left[\frac{\bar{r}_{1}}{\bar{a}_{11}}\right]+2 \bar{r}_{1} \omega:=B_{1}, \\
& x_{1}(k) \geq x_{1}\left(\eta_{1}\right)-\sum_{s=0}^{\omega-1}\left|x_{1}(s+1)-x_{1}(s)\right| \\
& \geq \ln \left[\frac{\bar{r}_{1}-\bar{a}_{12} \sum_{l=0}^{+\infty} K_{12}(-l) \exp \left(B_{20}\right)}{\bar{a}_{13} \sum_{l=0}^{+\infty} K_{13}(-l)}\right. \\
&\left.-\frac{\bar{a}_{13} \sum_{l=0}^{+\infty} K_{13}(-l) \exp \left(B_{22}\right)}{\bar{a}_{13} \sum_{l=0}^{+\infty} K_{13}(-l)}\right] \\
&-2 \bar{r}_{1} \omega:=B_{23} .
\end{aligned}
$$

It follows from (76) that

$$
\max _{k \in I_{\omega}}\left\{x_{3}(k)\right\} \leq \max \left\{\left|B_{1}\right|,\left|B_{23}\right|\right\}:=B_{24} .
$$

(e) If $x_{3}\left(\eta_{3}\right) \geq x_{1}\left(\eta_{1}\right) \geq x_{2}\left(\eta_{2}\right)$, then it follows from (23) that

$$
\begin{aligned}
& {\left[\bar{a}_{11}+\bar{a}_{12} \sum_{l=0}^{+\infty} K_{12}(-l)+\bar{a}_{13} \sum_{l=0}^{+\infty} K_{13}(-l)\right]} \\
& \quad \times \exp \left(x_{3}\left(\eta_{3}\right)\right) \omega \geq \bar{r}_{1} \omega
\end{aligned}
$$

which leads to

$$
x_{3}\left(\eta_{3}\right)>\ln \left[\frac{\bar{r}_{1}}{\bar{a}_{11}+\bar{a}_{12} \sum_{l=0}^{+\infty} K_{12}(-l)+\bar{a}_{13} \sum_{l=0}^{+\infty} K_{13}(-l)}\right] .
$$

It follows from (29), (79), and Lemma 2 that

$$
\begin{aligned}
x_{3}(k) \leq & x_{3}\left(\xi_{3}\right)+\sum_{s=0}^{\omega-1}\left|x_{3}(s+1)-x_{3}(s)\right| \\
\leq & \ln \left[\frac{\bar{r}_{3}}{\bar{a}_{33}}\right]+2 \bar{r}_{3} \omega:=B_{7}, \\
x_{3}(k) \geq & x_{3}\left(\eta_{3}\right)-\sum_{s=0}^{\omega-1}\left|x_{3}(s+1)-x_{3}(s)\right| \\
\geq & \ln \left[\frac{\bar{r}_{1}}{\bar{a}_{11}+\bar{a}_{12} \sum_{l=0}^{+\infty} K_{12}(-l)+\bar{a}_{13} \sum_{l=0}^{+\infty} K_{13}(-l)}\right] \\
& -2 \bar{r}_{3} \omega:=B_{32} .
\end{aligned}
$$

By (80), we derive

$$
\max _{k \in I_{\omega}}\left\{x_{3}(k)\right\} \leq \max \left\{\left|B_{7}\right|,\left|B_{32}\right|\right\}:=B_{33} .
$$

From (23) and (33), we obtain that

$$
\begin{aligned}
{\left[\bar{a}_{11}\right.} & \left.+\bar{a}_{12} \sum_{l=0}^{+\infty} K_{12}(-l)\right] \omega \exp \left(x_{1}\left(\eta_{1}\right)\right) \\
& +\bar{a}_{13} \sum_{l=0}^{+\infty} K_{13}(-l) \omega \exp \left(B_{33}\right) \geq \bar{r}_{1} \omega .
\end{aligned}
$$

Then

$$
x_{1}\left(\eta_{1}\right) \geq \ln \left[\frac{\bar{r}_{1}-\bar{a}_{13} \sum_{l=0}^{+\infty} K_{13}(-l) \exp \left(B_{33}\right)}{\bar{a}_{11}+\bar{a}_{12} \sum_{l=0}^{+\infty} K_{12}(-l)}\right] .
$$


Thus by (29), (83), and Lemma 2, we get

$$
\begin{aligned}
x_{1}(k) \leq & x_{1}\left(\xi_{1}\right)+\sum_{s=0}^{\omega-1}\left|x_{1}(s+1)-x_{1}(s)\right| \\
\leq & \ln \left[\frac{\bar{r}_{1}}{\bar{a}_{11}}\right]+2 \bar{r}_{1} \omega:=B_{1}, \\
x_{1}(k) \geq & x_{1}\left(\eta_{1}\right)-\sum_{s=0}^{\omega-1}\left|x_{1}(s+1)-x_{1}(s)\right| \\
\geq & \ln \left[\frac{\bar{r}_{1}-\bar{a}_{13} \sum_{l=0}^{+\infty} K_{13}(-l) \exp \left(B_{33}\right)}{\bar{a}_{11}+\bar{a}_{12} \sum_{l=0}^{+\infty} K_{12}(-l)}\right] \\
& -2 \bar{r}_{1} \omega:=B_{34} .
\end{aligned}
$$

It follows from (83) and (84) that

$$
\max _{k \in I_{\omega}}\left\{x_{1}(k)\right\} \leq \max \left\{\left|B_{1}\right|,\left|B_{34}\right|\right\}:=B_{35} .
$$

In view of (83), (84), and (23), we get

$$
\begin{gathered}
\bar{a}_{11} \omega \exp \left(B_{35}\right)+\bar{a}_{12} \omega \sum_{l=0}^{+\infty} K_{12}(-l) \exp \left(x_{2}\left(\eta_{2}\right)\right) \\
+\bar{a}_{13} \omega \sum_{l=0}^{+\infty} K_{13}(-l) \exp \left(B_{33}\right) \geq \bar{r}_{1} \omega
\end{gathered}
$$

Then

$$
\begin{aligned}
& x_{2}\left(\eta_{2}\right) \\
& \qquad \geq \ln \left[\frac{\bar{r}_{1}-\bar{a}_{11} \exp \left(B_{1}\right)-\bar{a}_{13} \sum_{l=0}^{+\infty} K_{13}(-l) \exp \left(B_{33}\right)}{\bar{a}_{13} \sum_{l=0}^{+\infty} K_{13}(-l)}\right] .
\end{aligned}
$$

Thus by (29), (88), and Lemma 2, we get

$$
\begin{aligned}
x_{2}(k) & \leq x_{2}\left(\xi_{2}\right)+\sum_{s=0}^{\omega-1}\left|x_{2}(s+1)-x_{2}(s)\right| \\
& \leq \ln \left[\frac{\bar{r}_{2}}{\bar{a}_{22}}\right]+2 \bar{r}_{2} \omega:=B_{4}, \\
x_{2}(k) \geq & x_{2}\left(\eta_{3}\right)-\sum_{s=0}^{\omega-1}\left|x_{2}(s+1)-x_{2}(s)\right| \\
\geq & \ln \left[\frac{\bar{r}_{1}-\bar{a}_{11} \exp \left(B_{4}\right)-\bar{a}_{13} \sum_{l=0}^{+\infty} K_{13}(-l) \exp \left(B_{33}\right)}{\bar{a}_{13} \sum_{l=0}^{+\infty} K_{13}(-l)}\right] \\
& -2 \bar{r}_{1} \omega:=B_{36} .
\end{aligned}
$$

It follows from (89) that

$$
\max _{k \in I_{\omega}}\left\{x_{2}(k)\right\} \leq \max \left\{\left|B_{4}\right|,\left|B_{36}\right|\right\}:=B_{37} .
$$

(f) If $x_{3}\left(\eta_{3}\right) \geq x_{2}\left(\eta_{2}\right) \geq x_{1}\left(\eta_{1}\right)$, then it follows from (23) that

$$
\begin{aligned}
& {\left[\bar{a}_{11}+\bar{a}_{12} \sum_{l=0}^{+\infty} K_{12}(-l)+\bar{a}_{13} \sum_{l=0}^{+\infty} K_{13}(-l)\right]} \\
& \quad \times \exp \left(x_{3}\left(\eta_{3}\right)\right) \omega \geq \bar{r}_{1} \omega
\end{aligned}
$$

which leads to

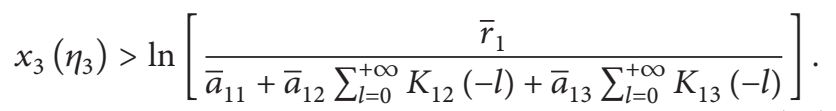

It follows from (29), (92), and Lemma 2 that

$$
\begin{aligned}
x_{3}(k) \leq & x_{3}\left(\xi_{3}\right)+\sum_{s=0}^{\omega-1}\left|x_{3}(s+1)-x_{3}(s)\right| \\
\leq & \ln \left[\frac{\bar{r}_{3}}{\bar{a}_{33}}\right]+2 \bar{r}_{3} \omega:=B_{7}, \\
x_{3}(k) \geq & x_{3}\left(\eta_{3}\right)-\sum_{s=0}^{\omega-1}\left|x_{3}(s+1)-x_{3}(s)\right| \\
\geq & \ln \left[\frac{\bar{r}_{1}}{\bar{a}_{11}+\bar{a}_{12} \sum_{l=0}^{+\infty} K_{12}(-l)+\bar{a}_{13} \sum_{l=0}^{+\infty} K_{13}(-l)}\right] \\
& -2 \bar{r}_{3} \omega:=B_{38} .
\end{aligned}
$$

By (93), we derive

$$
\max _{k \in I_{\omega}}\left\{x_{3}(k)\right\}<\max \left\{\left|B_{7}\right|,\left|B_{38}\right|\right\}:=B_{39} .
$$

From (23) and (94), we obtain that

$$
\begin{aligned}
{\left[\bar{a}_{11}\right.} & \left.+\bar{a}_{12} \sum_{l=0}^{+\infty} K_{12}(-l)\right] \omega \exp \left(x_{2}\left(\eta_{2}\right)\right) \\
& +\bar{a}_{13} \sum_{l=0}^{+\infty} K_{13}(-l) \omega \exp \left(B_{39}\right) \geq \bar{r}_{1} \omega .
\end{aligned}
$$

Then

$$
x_{2}\left(\eta_{2}\right) \geq \ln \left[\frac{\bar{r}_{1}-\bar{a}_{13} \sum_{l=0}^{+\infty} K_{13}(-l) \exp \left(B_{39}\right)}{\bar{a}_{11}+\bar{a}_{12} \sum_{l=0}^{+\infty} K_{12}(-l)}\right] .
$$


Thus by (29), (96), and Lemma 2, we get

$$
\begin{aligned}
& x_{2}(k) \leq x_{2}\left(\xi_{2}\right)+\sum_{s=0}^{\omega-1}\left|x_{2}(s+1)-x_{2}(s)\right| \\
& \leq \ln \left[\frac{\bar{r}_{2}}{\bar{a}_{22}}\right]+2 \bar{r}_{2} \omega:=B_{4}, \\
& x_{2}(k) \geq x_{2}\left(\eta_{2}\right)-\sum_{s=0}^{\omega-1}\left|x_{1}(s+1)-x_{1}(s)\right| \\
& \geq \ln \left[\frac{\bar{r}_{1}-\bar{a}_{13} \sum_{l=0}^{+\infty} K_{13}(-l) \exp \left(B_{39}\right)}{\bar{a}_{11}+\bar{a}_{12} \sum_{l=0}^{+\infty} K_{12}(-l)}\right] \\
& \quad-2 \bar{r}_{2} \omega:=B_{40} .
\end{aligned}
$$

It follows from (97) that

$$
\max _{k \in I_{\omega}}\left\{x_{2}(k)\right\} \leq \max \left\{\left|B_{4}\right|,\left|B_{40}\right|\right\}:=B_{41} .
$$

In view of (94), (98), and (23), we get

$$
\begin{gathered}
\bar{a}_{11} \omega \exp \left(x_{1}\left(\eta_{1}\right)\right)+\bar{a}_{12} \omega \sum_{l=0}^{+\infty} K_{12}(-l) \exp \left(B_{39}\right) \\
+\bar{a}_{13} \omega \sum_{l=0}^{+\infty} K_{13}(-l) \exp \left(B_{41}\right) \geq \bar{r}_{1} \omega .
\end{gathered}
$$

Then

$$
\begin{array}{r}
x_{1}\left(\eta_{1}\right) \geq \ln \left[\frac{\bar{r}_{1}-\bar{a}_{12} \sum_{l=0}^{+\infty} K_{12}(-l) \exp \left(B_{39}\right)}{\bar{a}_{11}}\right. \\
\left.-\frac{\bar{a}_{13} \sum_{l=0}^{+\infty} K_{13}(-l) \exp \left(B_{41}\right)}{\bar{a}_{11}}\right] .
\end{array}
$$

Thus by (29), (100), and Lemma 2, we get

$$
\begin{aligned}
x_{1}(k) & \leq x_{1}\left(\xi_{1}\right)+\sum_{s=0}^{\omega-1}\left|x_{1}(s+1)-x_{1}(s)\right| \\
& \leq \ln \left[\frac{\bar{r}_{1}}{\bar{a}_{11}}\right]+2 \bar{r}_{1} \omega:=B_{1},
\end{aligned}
$$

$$
\begin{aligned}
& x_{1}(k) \geq x_{1}\left(\eta_{1}\right)-\sum_{s=0}^{\omega-1}\left|x_{1}(s+1)-x_{1}(s)\right| \\
& \geq \ln \left[\frac{\bar{r}_{1}-\bar{a}_{12} \sum_{l=0}^{+\infty} K_{12}(-l) \exp \left(B_{39}\right)}{\bar{a}_{11}}\right. \\
&\left.-\frac{\bar{a}_{13} \sum_{l=0}^{+\infty} K_{13}(-l) \exp \left(B_{41}\right)}{\bar{a}_{11}}\right] \\
&-2 \bar{r}_{1} \omega:=B_{42} .
\end{aligned}
$$

It follows from (101) that

$$
\max _{k \in I_{\omega}}\left\{x_{2}(k)\right\} \leq \max \left\{\left|B_{1}\right|,\left|B_{42}\right|\right\}:=B_{43} .
$$

Obviously, $B_{i}(i=1,2, \ldots, 43)$ are independent of $\lambda \in(0,1)$. Take $M=\max \left\{B_{3}, B_{6}, B_{8}, B_{11}, B_{12}, B_{13}, B_{15}, B_{17}, B_{19}, B_{20}\right.$, $\left.B_{22}, B_{24}, B_{33}, B_{35}, B_{37}, B_{39}, B_{40}, B_{43},\right\}+B_{0}$, where $B_{0}$ is taken sufficiently large such that $\max \left\{\left|\ln \left\{x_{1}^{*}\right\}\right|,\left|\ln \left\{x_{2}^{*}\right\}\right|,\left|\ln \left\{x_{3}^{*}\right\}\right|\right\}<$ $B_{0}$, where $\left(x_{1}^{*}, x_{2}^{*}, x_{3}^{*}\right)^{T}$ is the unique positive solution of (11). Now we have proved that any solution $z=\{z(k)\}=$ $\left\{\left(x_{1}(k), x_{2}(k), x_{3}(k)\right)^{T}\right\}$ of (22) in $X$ satisfies $\|z\|<M, k \in Z$.

Let $\Omega:=\{z=\{z(k)\} \in X:\|z\|<M\}$, then it is easy to see that $\Omega$ is an open, bounded set in $X$ and verifies requirement (a) of Lemma 1. When $z \in \partial \Omega \cap \operatorname{Ker} L, z=\left\{\left(x_{1}, x_{2}, x_{3}\right)^{T}\right\}$ is a constant vector in $R^{3}$ with $\|z\|=\max \left\{\left|x_{1}\right|,\left|x_{2}\right|,\left|x_{3}\right|\right\}=M$. Then

$Q N z=$

$$
\begin{aligned}
& \left(\begin{array}{l}
\bar{r}_{1}-\bar{a}_{11} \exp \left(x_{1}\right)-\bar{a}_{12} \sum_{l=0}^{+\infty} K_{12}(-l) \exp \left(x_{2}\right)-\bar{a}_{13} \sum_{l=0}^{+\infty} K_{13}(-l) \exp \left(x_{3}\right) \\
\bar{r}_{2}-\bar{a}_{22} \exp \left(x_{2}\right)-\bar{a}_{21} \sum_{l=0}^{+\infty} K_{21}(-l) \exp \left(x_{1}\right)-\bar{a}_{23} \sum_{l=0}^{+\infty} K_{23}(-l) \exp \left(x_{3}\right) \\
\bar{r}_{3}-\bar{a}_{33} \exp \left(x_{3}\right)-\bar{a}_{31} \sum_{l=0}^{+\infty} K_{31}(-l) \exp \left(x_{1}\right)-\bar{a}_{32} \sum_{l=0}^{+\infty} K_{32}(-l) \exp \left(x_{2}\right)
\end{array}\right) \\
& \neq 0 .
\end{aligned}
$$

Now let us consider homotopic $\phi\left(x_{1}, x_{2}, x_{3} \mu\right)=\mu Q N z+(1-$ $\mu) G z, \mu \in[0,1]$, where

$$
G z=\left(\begin{array}{l}
\bar{r}_{1}-\bar{a}_{11} \exp \left(x_{1}\right) \\
\bar{r}_{2}-\bar{a}_{22} \exp \left(x_{2}\right) \\
\bar{r}_{3}-\bar{a}_{33} \exp \left(x_{3}\right)
\end{array}\right) .
$$

Letting $J$ be the identity mapping and by direct calculation, we get

$$
\begin{aligned}
& \operatorname{deg}\left\{\operatorname{JQN}\left(x_{1}, x_{2}, x_{3}\right)^{T} ; \Omega \bigcap \operatorname{ker} L ; 0\right\} \\
& =\operatorname{deg}\left\{Q N\left(x_{1}, x_{2}, x_{3}\right)^{T} ; \Omega \bigcap \operatorname{ker} L ; 0\right\} \\
& =\operatorname{deg}\left\{\phi\left(x_{1}, x_{2}, x_{3}, 1\right) ; \Omega \bigcap \operatorname{ker} L ; 0\right\} \\
& =\operatorname{deg}\left\{\phi\left(x_{1}, x_{2}, x_{3}, 0\right) ; \Omega \bigcap \operatorname{ker} L ; 0\right\}
\end{aligned}
$$




$$
\begin{aligned}
& =\operatorname{sign}\left\{\operatorname{det}\left(\begin{array}{ccc}
-\bar{a}_{11} \exp \left(x_{1}^{*}\right) & 0 & 0 \\
0 & -\bar{a}_{22} \exp \left(x_{2}^{*}\right) & 0 \\
0 & 0 & -\bar{a}_{33} \exp \left(x_{3}^{*}\right)
\end{array}\right)\right\} \\
& =\operatorname{sign}\left\{-\bar{a}_{11} \bar{a}_{22} \bar{a}_{33} \exp \left(x_{1}^{*}+x_{2}^{*}+x_{3}^{*}\right)\right\}=-1 \neq 0 .
\end{aligned}
$$

By now, we have proved that $\Omega$ verifies all requirements of Lemma 1, then it follows that $L z=N z$ has at least one solution in $\operatorname{Dom} L \cap \bar{\Omega}$; that is, to say, (11) has at least one $\omega$ periodic solution in $\operatorname{Dom} L \cap \bar{\Omega}$ say $z^{*}=\left\{z^{*}(k)\right\}=$ $\left\{\left(x_{1}^{*}(k), x_{2}^{*}(k), x_{3}^{*}(k)\right)^{T}\right\}$. Let $\bar{x}_{1}^{*}(k)=\exp \left\{x_{1}^{*}(k)\right\}, \bar{x}_{2}^{*}(k)=$ $\exp \left\{x_{2}^{*}(k)\right\}$, and $\bar{x}_{3}^{*}(k)=\exp \left\{x_{3}^{*}(k)\right\}$; then by Lemma 3 we know that $\left.\bar{z}^{*}=\left\{\bar{x}^{*}(k)\right\}=\left\{\bar{x}_{1}^{*}(k), \bar{x}_{2}^{*}(k), \bar{x}_{3}^{*}(k)\right)^{T}\right\}$ is an $\omega$ periodic solution of system (7) with strictly positive components. The proof is complete.

\section{Acknowledgments}

This work is supported by the National Natural Science Foundation of China (nos. 11261010 and no. 11101126), the Soft Science and Technology Program of Guizhou Province (no. 2011LKC2030), the Natural Science and Technology Foundation of Guizhou Province (J[2012] 2100), the Governor Foundation of Guizhou Province ([2012] 53), and the Doctoral Foundation of Guizhou University of Finance and Economics (2010).

\section{References}

[1] Y. Kuang, Delay Differential Equations with Applications in Population Dynamics, vol. 191, Academic Press, Boston, Mass, USA, 1993.

[2] X. H. Tang and X. F. Zou, "On positive periodic solutions of Lotka-Volterra competition systems with deviating arguments," Proceedings of the American Mathematical Society, vol. 134, no. 10, pp. 2967-2974, 2006.

[3] X. Tang, D. Cao, and X. Zou, "Global attractivity of positive periodic solution to periodic Lotka-Volterra competition systems with pure delay," Journal of Differential Equations, vol. 228, no. 2, pp. 580-610, 2006.

[4] M. Bohner, M. Fan, and J. Zhang, "Existence of periodic solutions in predator-prey and competition dynamic systems," Nonlinear Analysis. Real World Applications, vol. 7, no. 5, pp. 1193-1204, 2006.

[5] C. V. Pao, "Global asymptotic stability of Lotka-Volterra competition systems with diffusion and time delays," Nonlinear Analysis. Real World Applications, vol. 5, no. 1, pp. 91-104, 2004.

[6] K. Gopalsamy and P. Weng, "Global attractivity in a competition system with feedback controls," Computers \& Mathematics with Applications, vol. 45, no. 4-5, pp. 665-676, 2003.

[7] X. Xiong and Z. Zhang, "Periodic solutions of a discrete twospecies competitive model with stage structure," Mathematical and Computer Modelling, vol. 48, no. 3-4, pp. 333-343, 2008.

[8] Z. Liu, J. Hui, and J. Wu, "Permanence and partial extinction in an impulsive delay competitive system with the effect of toxic substances," Journal of Mathematical Chemistry, vol. 46, no. 4, pp. 1213-1231, 2009.

[9] S. Ahmad and I. M. Stamova, "Asymptotic stability of an $N$ dimensional impulsive competitive system," Nonlinear Analysis. Real World Applications, vol. 8, no. 2, pp. 654-663, 2007.
[10] Y. Chen and Z. Zhou, "Stable periodic solution of a discrete periodic Lotka-Volterra competition system," Journal of Mathematical Analysis and Applications, vol. 277, no. 1, pp. 358-366, 2003.

[11] M. Zhu and S. Lu, "Existence and global attractivity of positive periodic solutions of competition systems," Journal of Applied Mathematics and Computing, vol. 37, no. 1-2, pp. 635-646, 2011.

[12] Y. G. Sun and S. H. Saker, "Positive periodic solutions of discrete three-level food-chain model of Holling type II," Applied Mathematics and Computation, vol. 180, no. 1, pp. 353-365, 2006.

[13] R. Xu, L. Chen, and F. Hao, "Periodic solutions of a discrete time Lotka-Volterra type food-chain model with delays," Applied Mathematics and Computation, vol. 171, no. 1, pp. 91-103, 2005.

[14] Y. Li, "Positive periodic solutions of a discrete mutualism model with time delays," International Journal of Mathematics and Mathematical Sciences, no. 4, pp. 499-506, 2005.

[15] L. Nie, Z. Teng, L. Hu, and J. Peng, "Existence and stability of periodic solution of a predator-prey model with statedependent impulsive effects," Mathematics and Computers in Simulation, vol. 79, no. 7, pp. 2122-2134, 2009.

[16] L.-L. Wang and W.-T. Li, "Periodic solutions and permanence for a delayed nonautonomous ratio-dependent predator-prey model with Holling type functional response," Journal of Computational and Applied Mathematics, vol. 162, no. 2, pp. 341-357, 2004.

[17] J. Wiener, "Differential equations with piecewise constant delays," in Trends in Theory and Practice of Nonlinear Differential Equations, vol. 90 of Lecture Notes in Pure and Applied Mathematics, pp. 547-552, Dekker, New York, NY, USA, 1984.

[18] B. Dai and J. Zou, "Periodic solutions of a discrete-time nonautonomous predator-prey system with the BeddingtonDeAngelis functional response," Journal of Applied Mathematics \& Computing, vol. 24, no. 1-2, pp. 127-139, 2007.

[19] W. Zhang, D. Zhu, and P. Bi, "Multiple positive periodic solutions of a delayed discrete predator-prey system with type IV functional responses," Applied Mathematics Letters of Rapid Publication, vol. 20, no. 10, pp. 1031-1038, 2007.

[20] M. Fan and K. Wang, "Periodic solutions of a discrete time nonautonomous ratio-dependent predator-prey system," Mathematical and Computer Modelling, vol. 35, no. 9-10, pp. 951-961, 2002.

[21] R. E. Gaines and J. L. Mawhin, Coincidence Degree, and Nonlinear Differential Equations, Springer, Berlin, Germany, 1997. 


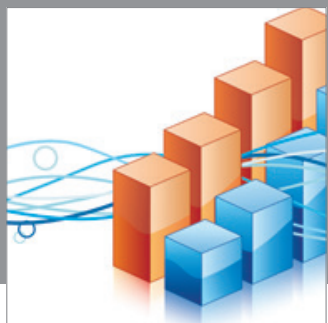

Advances in

Operations Research

mansans

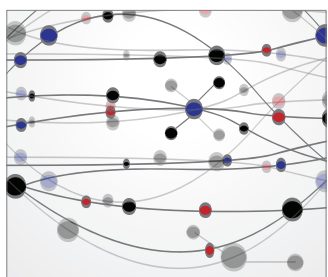

The Scientific World Journal
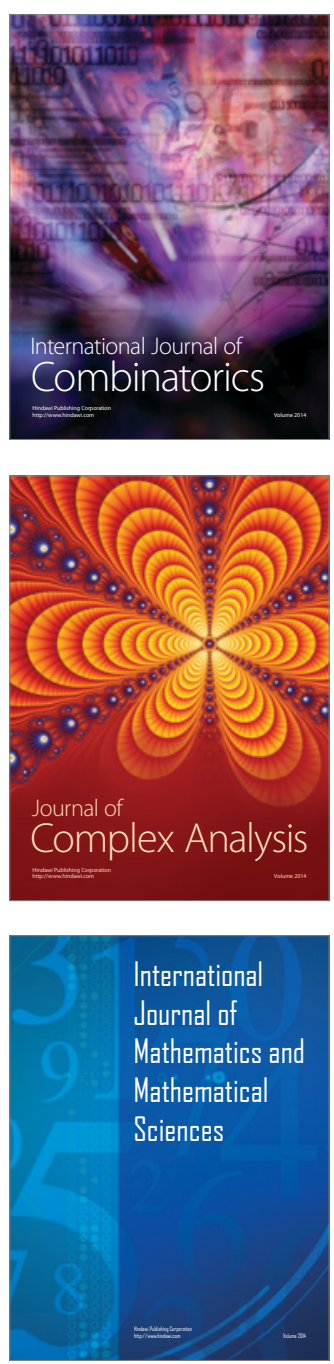
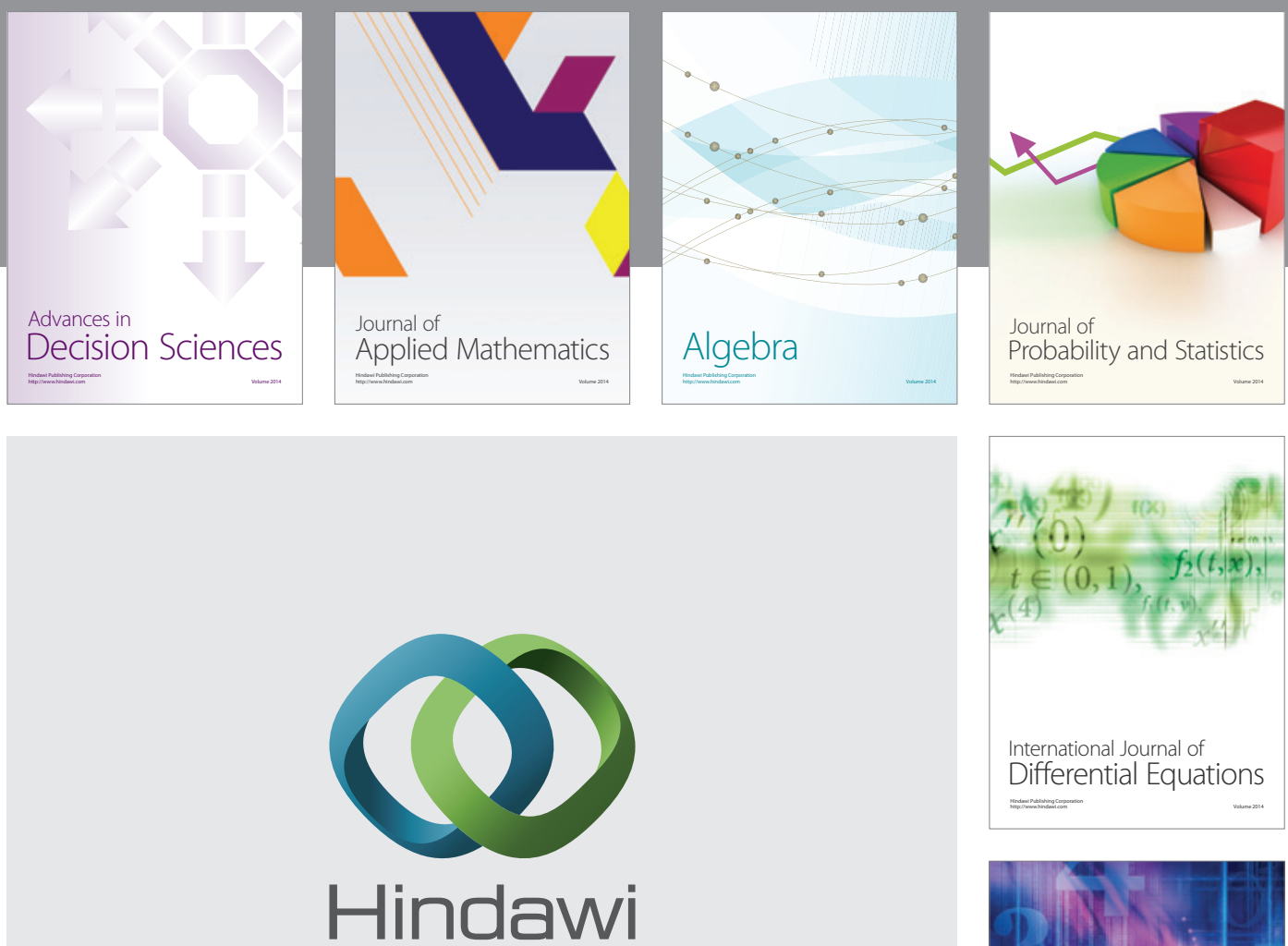

Submit your manuscripts at http://www.hindawi.com
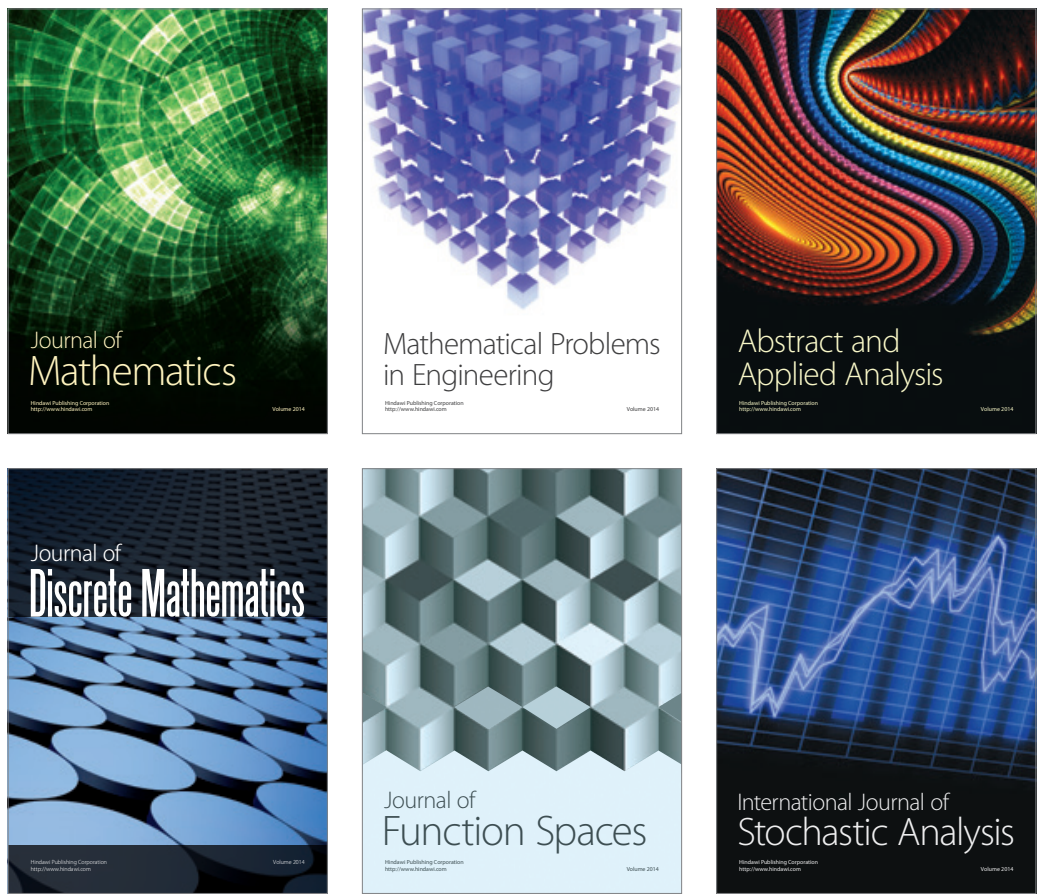

Journal of

Function Spaces

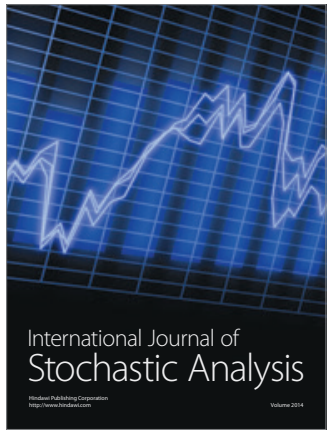

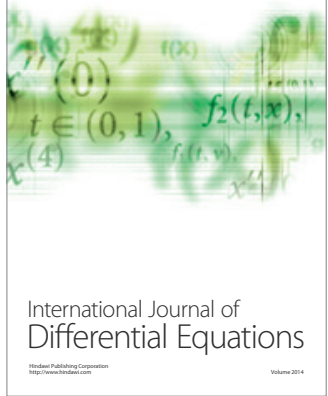
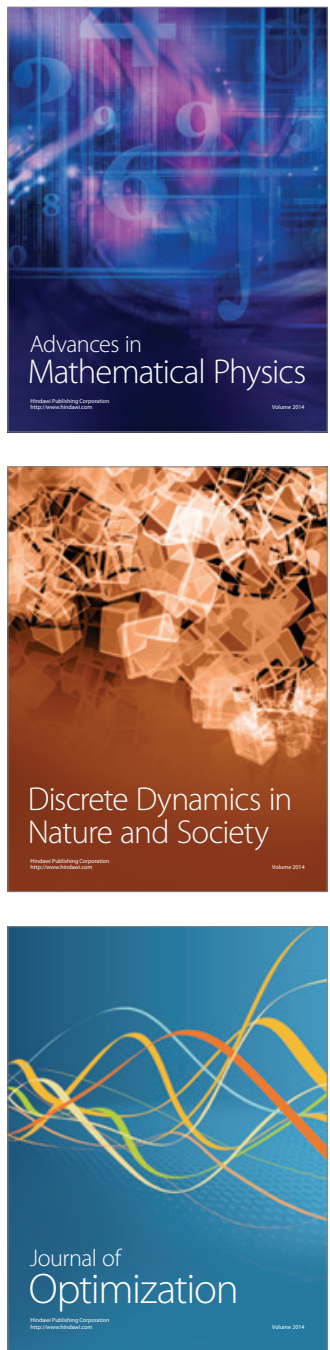